\title{
Metformin potentiates the anticancer activities of gemcitabine and cisplatin against cholangiocarcinoma cells in vitro and in vivo
}

\author{
HUA-QIANG ZHU ${ }^{1}$, JIN-BEN MA ${ }^{2}$, XIE SONG ${ }^{1}$, HENG-JUN GAO $^{1}$, CHAO-QUN MA $^{1}$, HONG CHANG ${ }^{1}$, \\ HONG-GUANG LI ${ }^{1}$, FANG-FENG LIU ${ }^{1}$, JUN LU ${ }^{1}$ and XU ZHOU ${ }^{1}$ \\ Departments of ${ }^{1}$ Hepatobiliary Surgery and ${ }^{2}$ Anesthesiology, Shandong Provincial Hospital \\ Affiliated to Shandong University, Jinan, Shandong 250014, P.R. China
}

Received May 4, 2016; Accepted June 14, 2016

DOI: $10.3892 / o r .2016 .5187$

\begin{abstract}
Metformin, an oral biguanide drug used to treat type 2 diabetes, has displayed anticancer activities in several types of cancer cells. The combination of gemcitabine and cisplatin is the standard chemotherapy regimen for cholangiocarcinoma, but its benefit is limited. The present study aimed to investigate whether metformin could enhance the activities of gemcitabine and cisplatin against cholangiocarcinoma, and the underlying mechanisms. Metformin inhibited the proliferation of human cholangiocarcinoma RBE and HCCC-9810 cells and induced cell cycle arrest at the G0/G1 phase by increasing the activation of AMP-activated protein kinase (AMPK) pathways. Metformin upregulated the expression of $\mathrm{p} 21^{\text {Waf } 1}$ and $\mathrm{p} 27^{\mathrm{kip}}$, and downregulated the expression of cyclin D1, a key protein required for cell cycle progression. The combination of gemcitabine and cisplatin inhibited the proliferation and induced the apoptosis of human cholangiocarcinoma cells by inducing the phosphorylation of AMPK, downregulating cyclin D1, and activating caspase-3. Administration of metformin enhanced the efficacy of gemcitabine and cisplatin to suppress the growth of cholangiocarcinoma tumors established in experimental models by inhibiting cell proliferation and inducing cell apoptosis through their effects on AMPK, cyclin D1 and caspase-3. Given that metformin has been used to treat type 2 diabetes patients for over half a century due to its superior safety profile, the results presented here indicate that metformin may be a potent agent for enhancing the efficacy of gemcitabine and cisplatin in the treatment of cholangiocarcinoma.
\end{abstract}

Correspondence to: Dr Xu Zhou, Department of Hepatobiliary Surgery, Shandong Provincial Hospital Affiliated to Shandong University, 9677 Jingshi Road, Jinan, Shandong 250014, P.R. China E-mail:xupzhou@sina.com

Key words: cholangiocarcinoma, metformin, gemcitabine, cisplatin

\section{Introduction}

Cholangiocarcinoma is the second most common type of primary liver cancer (1). Over $90 \%$ of cholangiocarcinoma patients have lost the opportunity for curative surgical resection at the time of diagnosis (1). The current standard chemotherapeutic regimen for advanced cholangiocarcinoma is systemic administration of gemcitabine and cisplatin, but the survival benefit is far from satisfactory. Cholangiocarcinoma patients have a poor prognosis with a median survival of approximately one year (2). Therefore, it is urgently required to seek therapeutic strategies that could enhance the efficacy of the current chemotherapy regimen for the treatment of cholangiocarcinoma.

In seeking potential agents, metformin (dimethybiguanide), an oral biguanide drug used to treat type 2 diabetes (3), had attracted our attention. It has long been recognized that insulin resistance is associated with the risk for the development of several types of human cancers (4). Moreover, the results of a cohort study indicate that the use of metformin is associated with a reduced incidence of cancer in patients with type 2 diabetes (5). The epidemiologic studies have led to investigations of metformin as an anticancer drug and the underlying mechanisms in cell culture and animal models. Metformin has been shown to inhibit cell proliferation, induce cell cycle arrest and promote apoptosis by activating the AMP-activated protein kinase (AMPK) pathways (6). AMPK is a central cellular energy sensor and a crucial factor in the interaction between metabolism and cancer (6). Activation of AMPK by metformin results in the inhibition of mammalian target of rapamycin (mTOR) signaling pathways and stimulation of the p53/p21 axis (7). Several studies have reported that metformin inhibits the growth of gastric cancer (7), leukemia (8), prostate (9) and esophageal cancer (10), and hepatocellular carcinoma cells (11). A recent study has demonstrated that metformin also exhibits an antitumor effect against cholangiocarcinoma cells (12). In addition, metformin was found to synergize with 5-fluorouracil, epirubicin and cyclophosphamide to suppress breast cancer cell growth (13), to enhance the efficacy of 5-fluorouracil to inhibit esophageal adenocarcinoma cell growth (14), and to sensitize intrahepatic cholangiocarcinoma cells to sorafenib, 5-fluorouracil and $\mathrm{As}_{2} \mathrm{O}_{3}$ (15). However, it is unknown whether metformin could also be used to strengthen the efficacy of 
gemcitabine and cisplatin, the standard chemotherapeutic regimen for cholangiocarcinoma.

\section{Materials and methods}

Cell culture. Human cholangiocarcinoma cell lines, RBE and HCCC-9810, were obtained from the Chinese Academy of Sciences Cell Bank (Shanghai, China). Cells were cultured at $37^{\circ} \mathrm{C}$ in Dulbecco's modified Eagle's medium (DMEM) (Gibco-BRL, Grand Island, NY, USA) supplemented with $10 \%$ fetal bovine serum.

Reagents and antibodies. Gemcitabine and cisplatin were provided by Jinan Trio PharmaTech Co., Ltd. (Jinan, China). Metformin was purchased from Sigma-Aldrich (Shanghai, China). Metformin, gemcitabine and cisplatin were dissolved in water to make stock solutions of 100,5 and $5 \mathrm{mM}$, respectively. Antibodies (Abs) against AMPK, phosphorylated AMPK (pAMPK and Thr172), caspase-3 and cyclin D1 were purchased from Cell Signaling Technology (Beverly, MA, USA). Abs against p2 $1^{\text {Waf1}}, \mathrm{p} 27^{\mathrm{kip} 1}$ and anti- $\beta$-actin were purchased from Santa Cruz Biotechnology (Dallas, TX, USA). An anti-Ki-67 Ab was purchased from Abcam Inc. (Cambridge, MA, USA).

Cell viability assay. The Cell Counting Kit-8 (CCK-8) (Dojindo Molecular Technologies, Inc., Beijing, China) was used to determine cell viability. Cells were seeded at $1 \times 10^{3}$ cells/well in 96-well plates. At different time points after treatments, the culture medium was replaced with $100 \mu 1$ of fresh medium containing $10 \mu \mathrm{l}$ of CCK-8 solution. Cells were further incubated for $2 \mathrm{~h}$ at $37^{\circ} \mathrm{C}$, and the optical density (OD) at $450 \mathrm{~nm}$ was measured. Untreated cells served as controls. The proliferation inhibition rate (\%) was calculated according to the formula: (Control OD value - experimental OD value/control OD value) x 100 .

Flow cytometry for assessing cell cycle distribution and apoptosis. Cells were seeded at $5 \times 10^{5}$ cells/well in 6 -well plates, incubated with treatment reagents, and harvested at indicated time points. The percentages of cells at the G2/M, S and G0/ G1 phases were determined using a cell cycle detection kit (BD Biosciences, Beijing, China) by a Beckman Coulter EPICS Altra II cytometer (Beckman Coulter, Brea, CA, USA). In addition, $1 \times 10^{5}$ cells were suspended in $100 \mu$ l binding buffer, incubated with $5 \mu \mathrm{l}$ of Annexin $\mathrm{V}$ and $5 \mu \mathrm{l}$ of propidium iodide (PI) for $15 \mathrm{~min}$ at room temperature in the dark, according to the manufacturer's instructions (BD Biosciences, San Jose, CA, USA). Then the cells were subjected to flow cytometry to measure the apoptosis rate (\%).

Measurement of caspase activity. The activity of caspase-3 in cell lysates was determined using the CaspACE ${ }^{\mathrm{TM}}$ Assay system (G7220; Promega Corp., Madison, WI, USA).

Western blot analysis. The western blotting methods have been previously described $(16,17)$. Cells were homogenized in protein lysate buffer, and debris was removed by centrifugation. Protein concentrations were determined. Lysates were resolved on sodium dodecyl sulfate-polyacrylamide gels and electrophoretically transferred to polyvinylidene difluoride membranes. The membranes were blocked, and incubated overnight with primary Abs, and subsequently with secondary horseradish peroxidase-conjugated Abs. They were developed with 5-bromo-4-chloro-3-indolyl phosphate (BCIP)/nitro blue tetrazolium (NBT) (Tiangen Biotech Co., Ltd., Beijing, China).

Animal experimental protocols. Animal experiments were performed according to the guidelines of the Animal Ethics Committee of Shandong University (Jinan, China). Six- to 8-week old male nude BALB/c mice (H-2b) were obtained from the Animal Research Center, Shandong University (China). Mice were maintained under specific pathogen-free conditions using a laminar airflow rack and had continuous free access to sterilized food and autoclaved water. RBE cells $\left(4 \times 10^{6}\right)$ were subcutaneously injected into the flanks of mice, which were monitored for appearance of palpable tumors. Tumor volume (V) was estimated by the formula: $\mathrm{V}=\pi / 6 \times a^{2} \times b$, where $a$ is the short axis, and $b$ the long axis. When tumors reached $\sim 150 \mathrm{~mm}^{3}$ in volume, mice were randomly assigned to 4 treatment groups (each group had 8 mice): control, metformin, gemcitabine/cisplatin and metformin plus gemcitabine/cisplatin. The mice in the metformin group received intraperitoneal injections of metformin at a dose of $2 \mathrm{mg} / \mathrm{kg}$ body weight 5 times a week for 28 days, while the mice in the control group received intraperitoneal injections of normal saline at the same volume and frequency as metformin solution. The mice in the gemcitabine/cisplatin group received biweekly intraperitoneal injections of gemcitabine at a dose of $100 \mathrm{mg} / \mathrm{kg}$ body weight, and weekly injections of cisplatin at a dose of $4 \mathrm{mg} / \mathrm{kg}$ body weight (18). The mice in the metformin plus gemcitabine/ cisplatin group received both injections of metformin and gemcitabine/cisplatin as described above. Thirty-five days after commencement of treatments, the mice were sacrificed. Samples were harvested for analysis.

In situ Ki-67 proliferation index. Formalin-fixed tumor specimens were transferred to $70 \%$ ethanol, and subsequently paraffin-embedded and sectioned. Tumor sections were blocked with $3 \%$ BSA for $2 \mathrm{~h}$, and incubated with an anti$\mathrm{Ki}-67 \mathrm{Ab}$ at $4{ }^{\circ} \mathrm{C}$ overnight. They were subsequently incubated for $30 \mathrm{~min}$ with the appropriate secondary Ab using the UltraSensitive TMS-P kit (Zhongshan Co., Ltd., Beijing, China), and immunoreactivity was developed with SIGMAFAST 3,3'-diaminobenzidine tetrahydrochloride (DAB) and $\mathrm{CoCl}_{2}$ enhancer tablets (Sigma-Aldrich, Shanghai, China). Sections were counterstained with hematoxylin, mounted and examined by microscopy. The Ki-67-positive cells were counted in 10 randomly selected $\mathrm{x} 400$ high-power fields under microscopy. The proliferation index was calculated according to the following formula: Number of Ki-67-positive cells/total cell count $\mathrm{x} 100 \%$.

In situ detection of apoptotic cells. Tumor sections were stained with the terminal deoxynucleotidyl transferase dUTP nick-end labeling (TUNEL) (Roche, Shanghai, China). The TUNEL-positive cells were counted in 20 randomly selected x200 high-power fields under microscopy. The apoptosis index was calculated according to the following formula: Number of apoptotic cells/total number of nucleated cells x $100 \%$. 

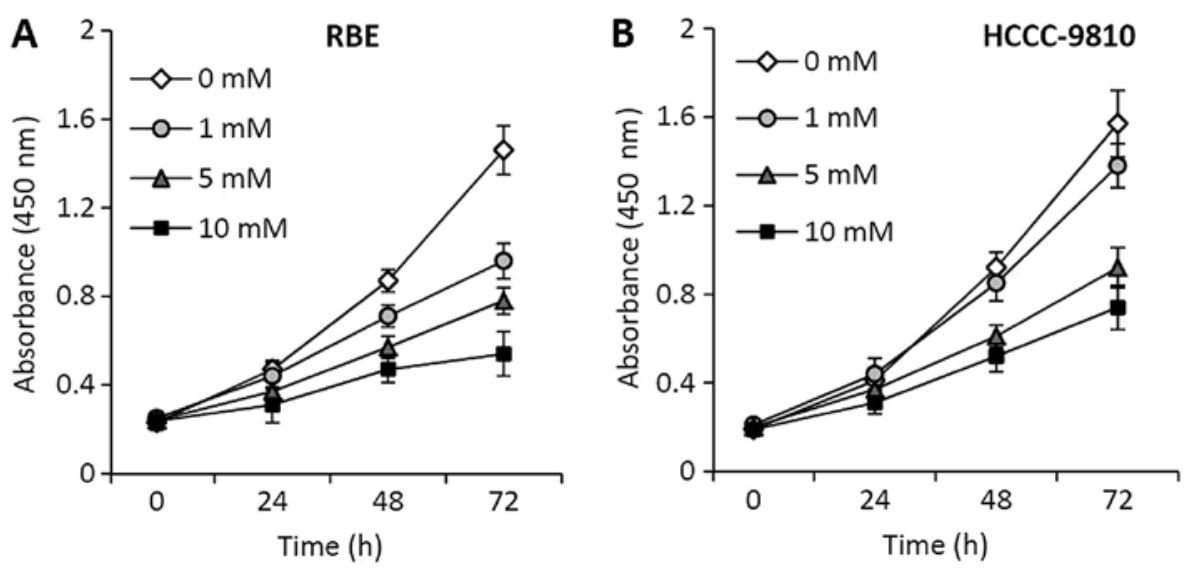

Figure 1. Metformin inhibits cell proliferation. (A) RBE and (B) HCCC-9810 cells were incubated with metformin at concentrations of $0,1,5$ or $10 \mathrm{mM}$ for 24 , 48 and $72 \mathrm{~h}$. Cell viability is represented by the optical density (OD) at $450 \mathrm{~nm}$.

Statistical analysis. The data are expressed as mean values \pm standard deviation. Comparisons were carried out with one-way analysis of variance (ANOVA) followed by Dunnet's test. $\mathrm{P}<0.05$ was considered to indicate a statistically significant result.

\section{Results}

Metformin inhibits the proliferation of cholangiocarcinoma cells. RBE and HCCC-9810 cells were incubated with metformin at various concentrations, and their viability was measured at 24,48 and $72 \mathrm{~h}$ after incubation. Metformin showed a strong inhibitory effect on the proliferation of RBE (Fig. 1A) and HCCC-9810 (Fig. 1B) cells in a time- and concentration-dependent manner.

Metformin induces cell cycle arrest. To further investigate the effects of metformin on cell proliferation, cell cycle progression was examined by flow cytometry. RBE cells were incubated with 0 or $5 \mathrm{mM}$ of metformin for 24 or $48 \mathrm{~h}$. As shown in Fig. 2A, metformin treatment induced an increasing number of cells that accumulated in the G0/G1 phase. Specifically, $58.7 \%$ of cells were arrested at the G0/G1 phase $24 \mathrm{~h}$ after treatment, while $70.2 \%$ of cells were arrested at the $\mathrm{G} 0 /$ G1 phase $48 \mathrm{~h}$ after treatment, compared with 42.5 and $49.4 \%$ for the untreated cells, respectively (Fig. 2A). This finding was accompanied by reductions in the percentages of cells in the $S$ and G2/M phases (Fig. 2B). The results suggest that metformin inhibits the cell cycle progression from $\mathrm{G} 0 / \mathrm{G} 1$ into $\mathrm{S}$ phases in cholangiocarcinoma cells.

We next investigated the expression of molecules involved in the action of metformin on cell proliferation by western blot analysis. As shown in Fig. 2C, metformin treatment induced an increased expression of pAMPK, while the expression of AMPK remained unchanged, indicating that metformin increased the activation of the AMPK pathway. The downstream factors, $\mathrm{p} 21^{\mathrm{Waf} 1}$ and $\mathrm{p} 27^{\mathrm{kip} 1}$, were also upregulated upon metformin treatment (Fig. 2C). Cyclin D1, a key protein required for the progression through the G1 phase of the cell cycle, was markedly downregulated in metformin-treated cells (Fig. 2C).
Gemcitabine and cisplatin inhibit cell proliferation and induce apoptosis. RBE and HCCC-9810 cells were incubated with the combination of gemcitabine and cisplatin (GC) for $48 \mathrm{~h}$. The concentrations of both gemcitabine and cisplatin ranged from 1.56 to $200 \mathrm{nM}$ based on independent dose finding experiments. Cell viability was measured and the proliferation inhibitory rates were calculated. As shown in Fig. 3, GC showed proliferation inhibitory effects against the two cell lines in a dose-dependent manner. With a simple linear regression analysis, the concentration of drugs resulting in $50 \%$ maximal proliferation inhibition $\left(\mathrm{IC}_{50}\right)$ was calculated to be $2.18 \mathrm{nM}$ for RBE cells, which were incubated with GC at $48 \mathrm{~h}$ (Fig. 3A); while the $\mathrm{IC}_{50}$ for HCCC-9810 cells was $3.54 \mathrm{nM}$ (Fig. 3B).

We next demonstrated whether GC could also induce cell apoptosis. As shown in Fig. 4A and B, GC induced apoptosis of RBE and HCCC-9810 cells in a dose-dependent manner. The representative histograms of flow cytometry showed that the apoptosis rates of RBE cells were $3.5,15.1$ or $25.6 \%$ when they underwent a 48-h incubation with GC at concentrations at $0 \mathrm{nM}$ (Fig. 4C), $1 \mathrm{nM}$ (Fig. 4D) or $4 \mathrm{nM}$ (Fig. 4E), respectively. The increased expression of cleaved caspase-3 (Fig. 4F) and activity of caspase-3 (Fig. 4G) correlated well with the cell apoptosis rates. The results indicate that GC induced the apoptosis of RBE cells through the caspase-dependent pathways in accordance with previous studies $(19,20)$.

Metformin increases the effects of gemcitabine and cisplatin on cholangiocarcinoma cells in vitro. We next examined whether metformin could enhance the activities of gemcitabine and cisplatin against cholangiocarcinoma cells. Based on the above results, a concentration of $\mathrm{GC}$ at $1 \mathrm{nM}$ below $\mathrm{IC}_{50}$ for both cell lines and a concentration of metformin at $5 \mathrm{mM}$ were selected for the following in vitro experiments. Cells were incubated with GC, metformin or their combination for $48 \mathrm{~h}$. Cell viability was measured and the proliferation inhibitory rates were calculated.

As shown in Fig. 5A (RBE) and B (HCCC-9810), a 48-h incubation with GC caused a $41.2 \%$ reduction in the viability of $\mathrm{RBE}$ cells, and a $28.9 \%$ reduction in the viability of HCCC-9810 cells, compared to the respective untreated cells. 
A

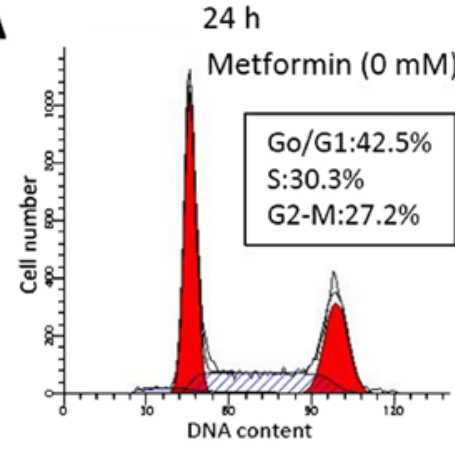

$48 \mathrm{~h}$

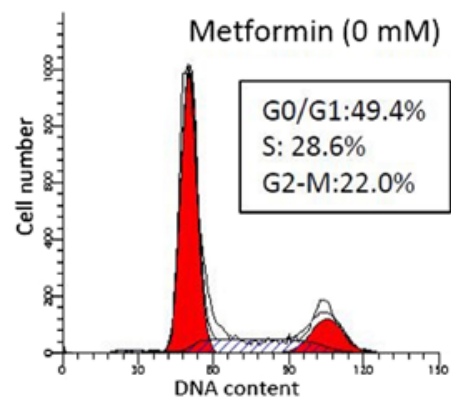

B

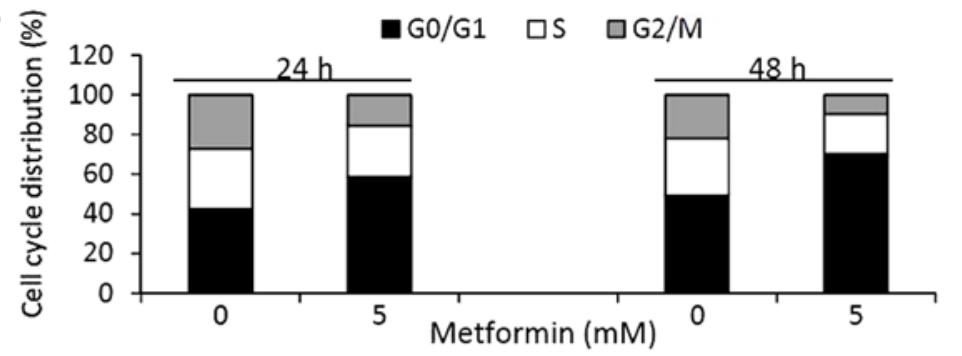

C

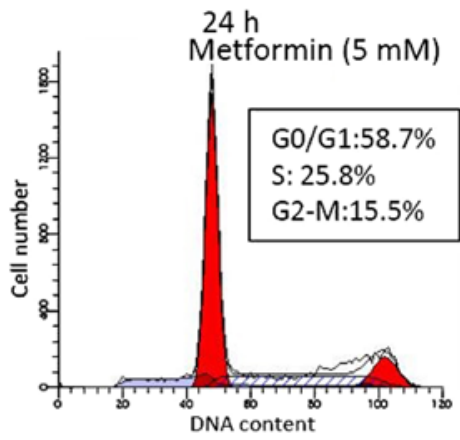

$48 \mathrm{~h}$

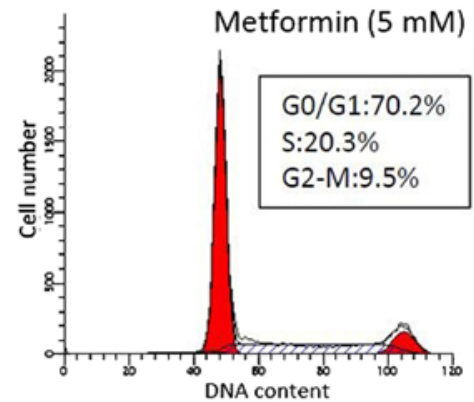

G0/G1:70.2\%

$\mathrm{S}: 20.3 \%$

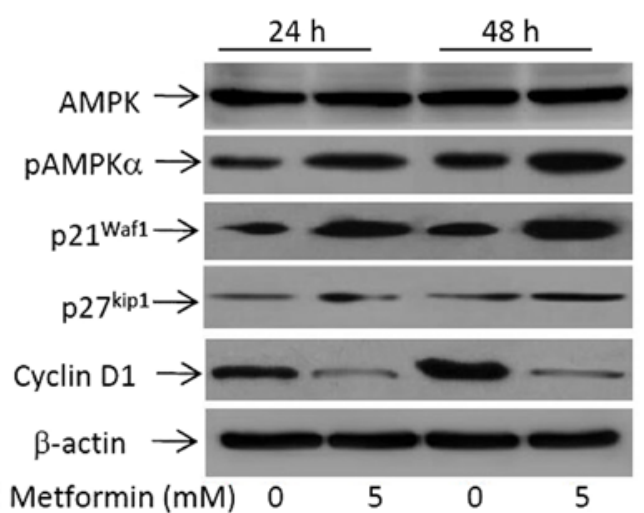

Figure 2. Metformin induces cell cycle arrest at the G0/G1 phase. (A) RBE cells were incubated with metformin (0 or $5 \mathrm{mM}$ ) for 24 or $48 \mathrm{~h}$, and then subjected to flow cytometry to measure cell cycle distribution. (B) The percentages of cells in the G0/G1, S and G2/M phases were plotted. (C) Lysates of the above cells were subjected to western blotting. $\beta$-actin served as an internal control.

Metformin therapy reduced the viability of RBE cells by $31.4 \%$, and that of HCCC-9810 cells by $24.7 \%$, compared to the respective untreated controls. However, the combination of GC and metformin further reduced the viability of RBE and HCCC-9810 cells by 63.2 and $50.6 \%$, respectively. To investigate whether the effects of GC and metformin are additive or synergistic, we calculated the value of the coefficient of drug interaction (CDI) as previously described (21). CDI was calculated according to the formula: $\mathrm{CDI}=\mathrm{AB} /(\mathrm{A} \times \mathrm{B})$, where $\mathrm{AB}$ is the ratio of the cell viability index of the combination group to the control group; A or B is the ratio of the viability index of the respective GC or metformin group to the control group. A value of CDI less than $(<)$, equal to $(=)$ or greater than $(>) 1$ indicates that the drugs are synergistic, additive or antagonistic, respectively (21). The values of CDI in the RBE and HCCC-9810 cells treated with GC and metformin were all $<1$, indicating that the two agents had synergistic effects in inhibiting the viability of the cells.

Similarly, GC and metformin also significantly increased the apoptosis of the RBE (Fig. 5C) and HCCC-9810 (Fig. 5D) 

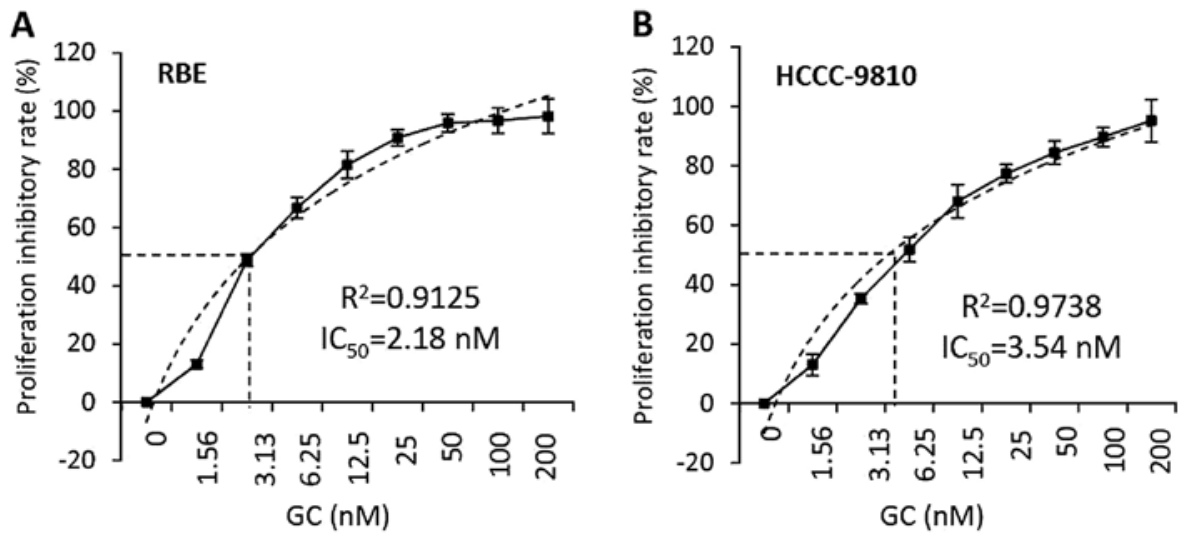

Figure 3. Combination of gemcitabine and cisplatin inhibits cell proliferation. (A) RBE and (B) HCCC-9810 cells were incubated with gemcitabine/cisplatin (GC) at concentrations ranging from 1.56 to $200 \mathrm{nM}$ for $48 \mathrm{~h}$. Untreated cells served as the controls. The viability of cells was assessed to calculate the proliferation inhibitory rate (\%). The linear regression was performed and the $\mathrm{R}^{2}$ value was calculated. The dotted curves show the trend of dose-dependent cell proliferation inhibition rates, and the dotted lines indicate drug concentrations, which resulted in $50 \%$ maximal proliferation inhibition (IC ${ }_{50}$ ) of the cells.

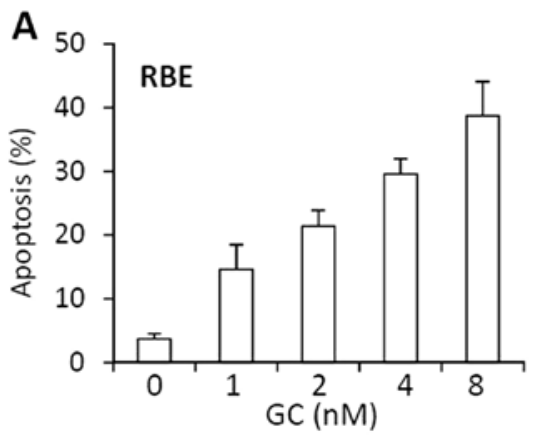

$\mathrm{C}$

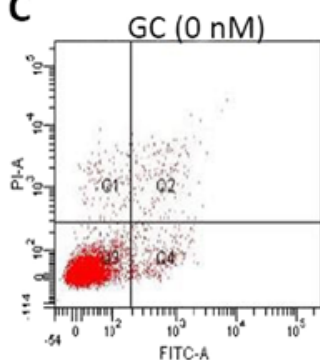

$\mathbf{F}$

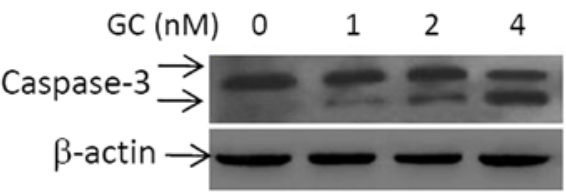

D
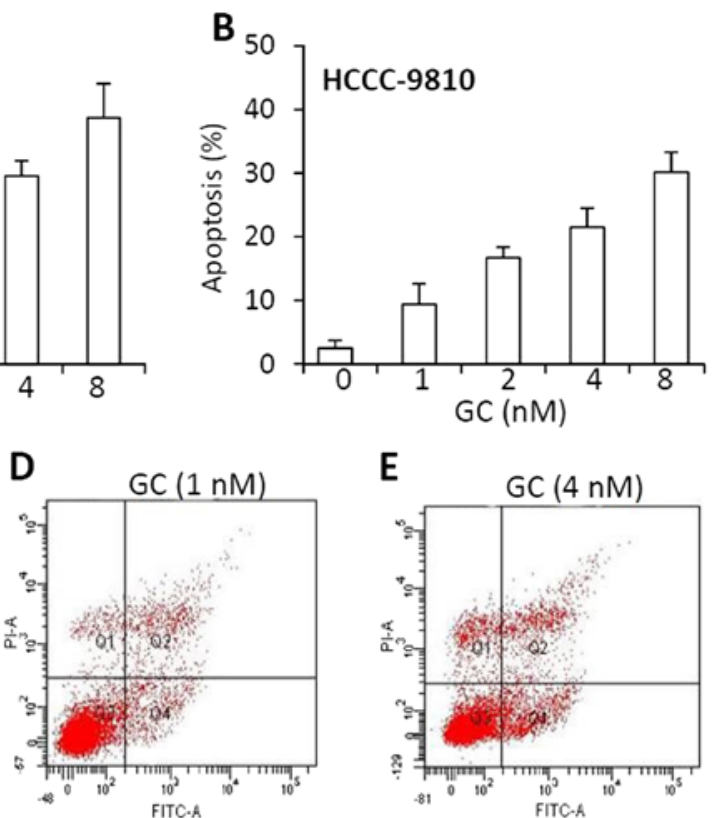

$\mathrm{E}$

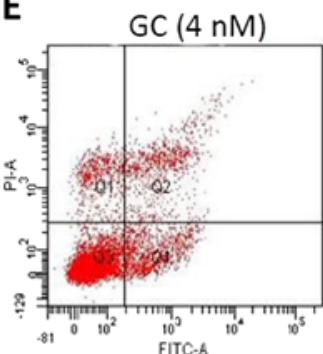

G

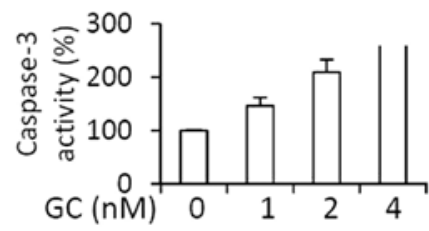

Figure 4. Combination of gemcitabine and cisplatin induces cell apoptosis. (A and B) RBE (A) and HCCC-9810 (B) cells were incubated with gemcitabine/cisplatin (GC) at concentrations of 1, 2, 4 or $8 \mathrm{nM}$ for $48 \mathrm{~h}$. Untreated cells served as the controls. Flow cytometry was performed to measure apoptosis rates. (C-E) Representative histograms were from cytometrically analyzed RBE cells incubated with GC at concentrations of (C) 0 nM, (D) $1 \mathrm{nM}$ or (E) $4 \mathrm{nM}$ for 48 h. (F. and G) Lysates of the above cells were subjected to (F) western blotting for caspase-3 or to (G) measurment of caspase-3 activity. $\beta$-actin served as an internal control for western blotting. Caspase-3 activity in untreated cells was defined as $100 \%$.

cells, and the combination of GC and metformin significantly induced higher apoptosis rates for both the RBE and HCCC-9810 cells than the rates noted in the untreated controls as well as for GC or metformin treatments.

The above RBE cells were further analyzed by western blot analysis to detect the alterations of key molecules involved in cell proliferation and apoptosis. GC, metformin or their combination had no significant effects on the expression of AMPK. GC and metformin upregulated the expression of pAMPK, and their combination further increased the expression of pAMPK (Fig. 5E). Both GC and metformin reduced the expression of cyclin D1, and the combination markedly decreased the expression of cyclin D1. Both GC and metformin significantly increased the cleavage of caspase-3 in the RBE 
A
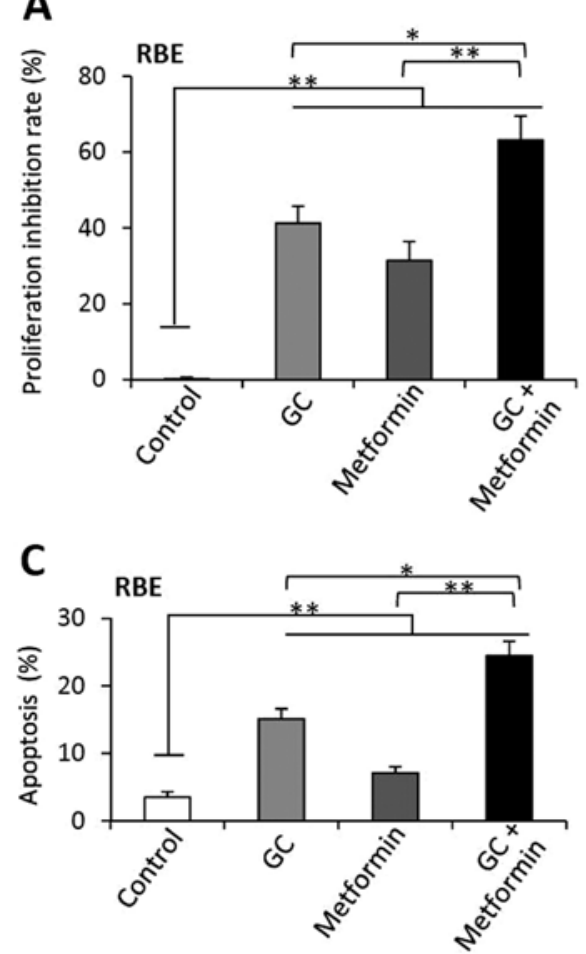

E

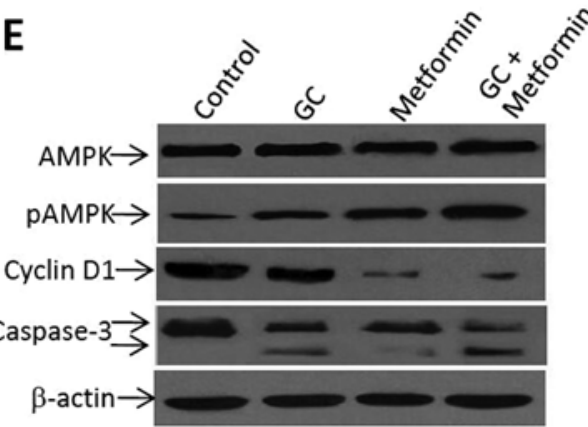

B

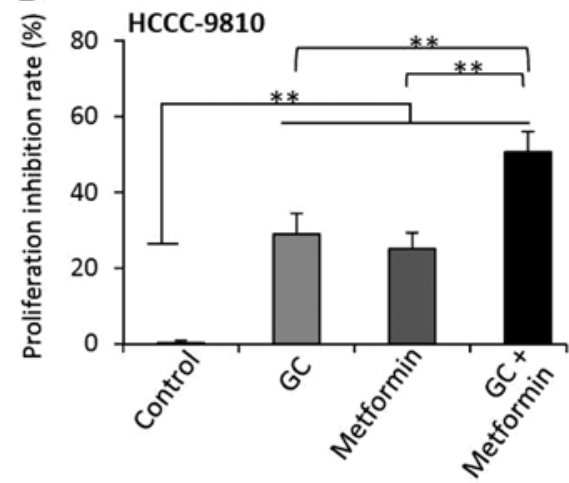

D
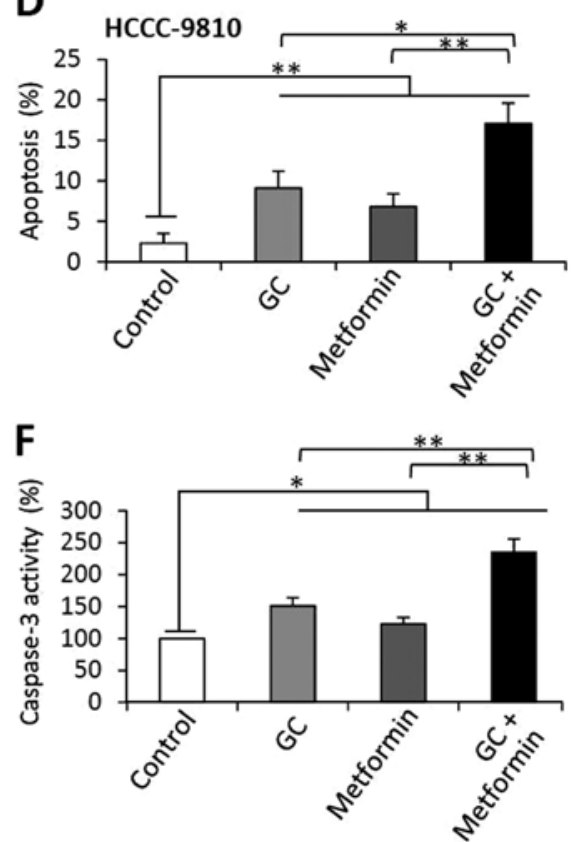

Figure 5. Metformin increases the effects of gemcitabine and cisplatin against cholangiocarcinoma cells. RBE and HCCC-9810 cells were treated with gemcitabine/cisplatin (GC) $(1 \mathrm{nM})$, metformin $(5 \mathrm{mM})$ or their combination. Untreated cells served as controls. (A and B) Cell viability was assessed to calculate the proliferation inhibitory rate $(\%)$. (C and D) Flow cytometry was performed to measure apoptosis rates. (E) Lysates of the above RBE cells were subjected to western blotting. $\beta$-actin served as an internal control. (F) The activity of caspase- 3 was measured in the above $\mathrm{RBE}$ cells; ${ }^{*} \mathrm{P}<0.05$ and ${ }^{* *} \mathrm{P}<0.001$ indicate a significant difference.

cells, and their combination increased to an even greater extent the cleavage of caspase- 3 in the RBE cells (Fig. 5E). The results of caspase-3 expression were supported by that of caspase-3 activities (Fig. 5F).

Metformin enhances the efficacy of gemcitabine and cisplatin to suppress tumor growth in vivo. Subcutaneous RBE tumors were established in the flanks of mice. When the tumors reached $\sim 150 \mathrm{~mm}^{3}$, the mice were randomly assigned to 4 treatment groups as described in Materials and methods. As shown in Fig. 6A, the control tumors grew markedly fast reaching $1613.5 \pm 109.1 \mathrm{~mm}^{3}$ in volume 35 days after treatment. In contrast, tumors in the gemcitabine/cisplatin-treated mice were significantly smaller, reaching only $758.4 \pm 81.2 \mathrm{~mm}^{3}$ in volume. Metformin therapy also significantly reduced tumor volume $\left(1015.3 \pm 72.6 \mathrm{~mm}^{3}\right)$, compared with the control tumors Tumors treated with the combination of gemcitabine/cisplatin and metformin reached only $349.8 \pm 57.1 \mathrm{~mm}^{3}$, and were significantly smaller than the volumes of the control tumors, and the tumors treated with either gemcitabine/cisplatin or metformin (Fig. 6A). Using the method mentioned above, we calculated the value of $\mathrm{CDI}$, which was 0.76 , indicating that gemcitabine/cisplatin and metformin had a synergistic effect in suppressing the growth of the RBE tumors.

Cell proliferation and apoptosis in situ. Tumor sections prepared from the above tumors were stained with an Ab which detects the cell proliferation marker Ki-67, or the TUNEL agent to detect apoptotic cells. There were fewer Ki-67positive and more apoptotic cells in the tumors treated with gemcitabine/cisplatin or metformin, when compared with the control tumors (Fig. 6B). The combination therapy resulted in even fewer Ki-67-positive and more apoptotic cells (Fig. 6B). Cells expressing Ki-67 were counted to calculate the proliferation index (Fig. 6C) and TUNEL-positive cells were counted to record the apoptosis index (Fig. 6D). As shown 
A

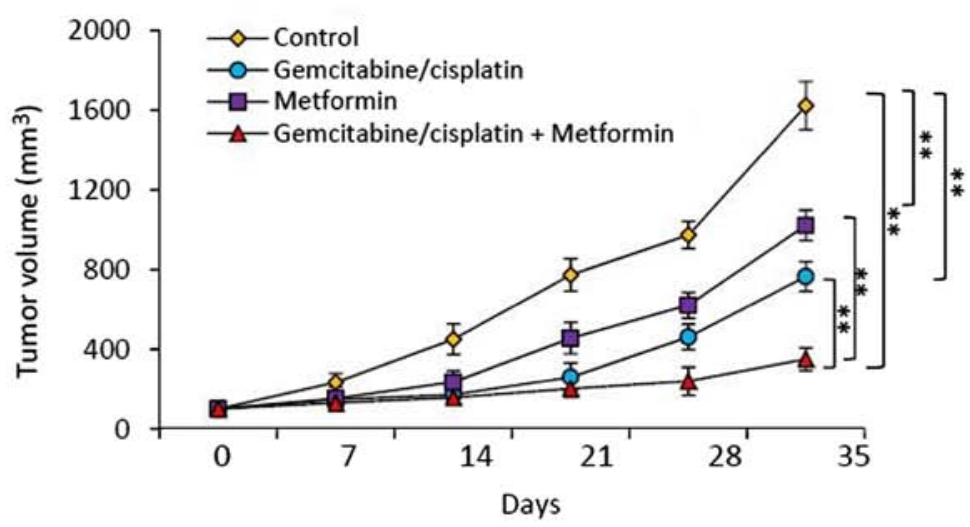

B

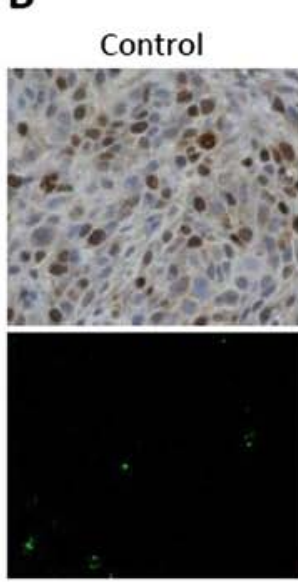

Gemcitabine /cisplatin
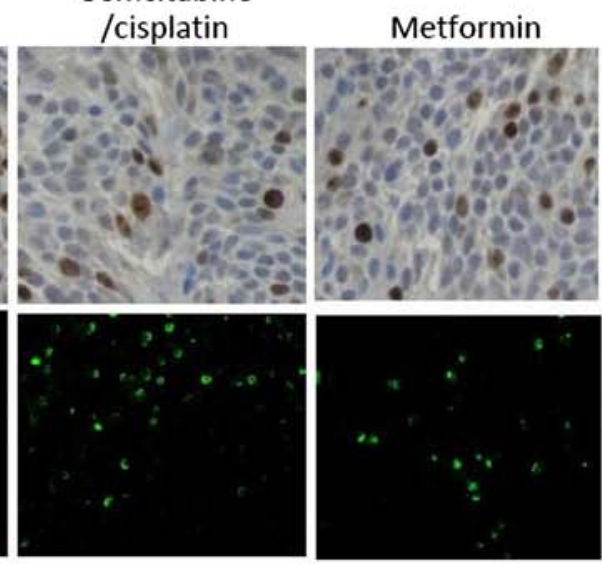

Gemcitabine

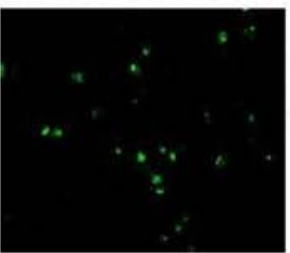

/cisplatin +

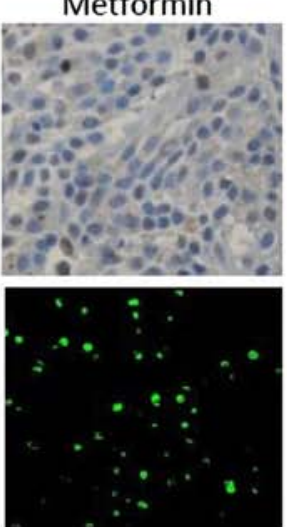

C

D
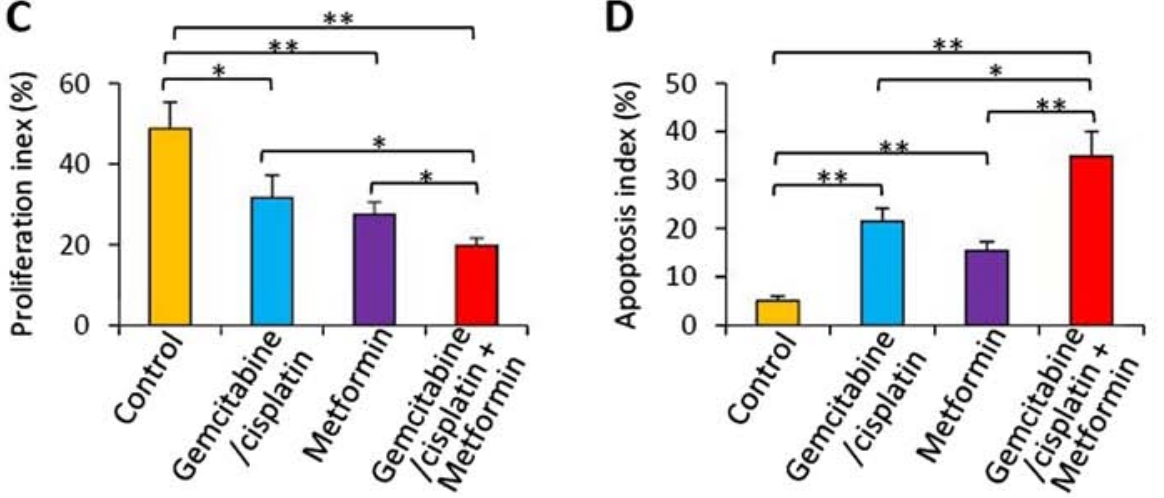

Figure 6. Tumor growth, cell proliferation and apoptosis in vivo. (A) RBE tumors were established subcutaneously in mice. When the tumors reached $\sim 150$ mm ${ }^{3}$ in volume, mice were randomly assigned to control, gemcitabine/cisplatin, metformin or gemcitabine/cisplatin + metformin groups, and treated as described in Materials and methods. The sizes $\left(\mathrm{mm}^{3}\right)$ of tumors were recorded. (B) Illustrated are representative tumor sections stained with an anti-Ki-67 Ab to detect proliferating cells (upper panel), or TUNEL agent to visualize apoptotic cells (lower panel). (C) Tumor cells expressing Ki-67 were counted to calculate the proliferation index or (D) TUNEL-positive cells to calculate the apoptosis index; ${ }^{*} \mathrm{P}<0.05$ and ${ }^{* *} \mathrm{P}<0.001$ indicate a significant difference.

in Fig. 6C, gemcitabine/cisplatin and metformin therapies resulted in a significant reduction in the proliferation index by 35.1 or $43.5 \%$, respectively, compared with the controls. The combination therapy resulted in a highly significant reduction in the proliferation index by $59.3 \%(\mathrm{P}<0.001)$ compared to the controls, and a significant reduction compared to either gemcitabine/cisplatin or metformin treatment (Fig. 6C). As shown in Fig. 6D, gemcitabine/cisplatin therapy significantly increased the apoptosis index by 3 -fold, and metformin therapy by 2 -fold, compared with the controls. The apoptosis index of the tumors treated with the combinational therapy was significantly higher by $>6$-fold than that of the control tumors, and also significantly higher than either gemcitabine/ cisplatin or metformin treatment (Fig. 6D).

\section{Discussion}

Cholangiocarcinoma, derived from the epithelial cells of biliary ducts, accounts for $\sim 3 \%$ of all gastrointestinal cancers. It is classified as intrahepatic, perihilar and extrahepatic cholangiocarcinoma (22). The incidence of intrahepatic cholangiocarcinoma has been increasing over the past 3 decades, and most patients have locally advanced or distal metastatic diseases at the time of presentation and lose the 
opportunity of curative surgery (10). The development of novel therapies, particularly molecular-targeted drugs, for cholangiocarcinoma is lagging behind other cancers (23). Conventional chemotherapy continues to play a critical role in the clinical management of cholangiocarcinoma. However, the most commonly used chemotherapy regimen, the combination of gemcitabine and cisplatin, only provides a very limited beneficial effect in prolonging the survival of patients (18). Therefore, the development of therapeutic strategies that enhance the efficacy of gemcitabine and cisplatin in combating cholangiocarcinoma is urgently needed.

Metformin is one of the most commonly used drugs for the management of type 2 diabetes worldwide. Metformin was recommended for the treatment of diabetes following a successful clinical trial in 1957 (24). Due to its superior safety profile, metformin has eventually become the first-line treatment for type 2 diabetes and it is now featured on the World Health Organization's list of essential medicines for both adults and children (WHO Model Lists of Essential Medicines 2015, World Health Organization). Notably, the therapeutic potential of metformin has recently extended far beyond its prescribed use as an anti-diabetic drug. There is a rapidly growing body of literature demonstrating an effective role for metformin in treating cancer and cardiovascular disease, delaying the aging process and modulating microbiota to promote health (25).

The AMPK signaling pathway has been widely studied in metabolic disorders and an increasing number of studies also suggest that it plays a potential role in cancer cell biology $(26,27)$. AMPK activation causes cell cycle arrest associated with stabilization of the cyclin-dependent kinase inhibitor $\mathrm{p} 21^{\text {Waf1 }}$ and $\mathrm{p} 27^{\mathrm{kip} 1}$ (28). The AMPK system is the key target for metformin, and activation of AMPK contributes largely to its anti-diabetic action (29). Metformin is able to induce the activation of AMPK in several types of cancer cells $(12-14,30,31)$. Upregulation of $\mathrm{p} 21^{\mathrm{Waf} 1}$ and $\mathrm{p} 27^{\mathrm{kip} 1}$ plays an important role in the inhibitory effects of metformin $(10,31)$. In agreement, the present study demonstrated that metformin treatment led to increased expression levels of p-AMPK $\alpha$, $\mathrm{p} 21^{\mathrm{Waf} 1}$ and $\mathrm{p} 27^{\mathrm{kip} 1}$, indicating activation of the AMPK pathway, in cholangiocarcinoma cells.

Metformin has been reported to induce cell cycle arrest at the G0/G1 phase by downregulating cyclin D1 in cells from gastric cancer (32), esophageal adenocarcinoma (10), hepatocellular carcinoma (33), as well as cholangiocarcinoma (12). The present results have shown that a large proportion of metformin-treated cells were arrested at the G0/G1 phase. Consistently, cyclin D1, a key protein required for progression through the G1 phase of the cell cycle (34), was markedly downregulated following metformin treatment.

Given the fact that metformin exhibits anticancer activities with a superior safety profile, the combination of metformin with cytotoxic chemotherapeutic agents is expected to be a promising strategy for cancer treatment. Diabetic patients with breast cancer taking metformin and undergoing neoadjuvant chemotherapy had a 3-fold higher pathologic complete response rate than those not taking metformin (35). An epidemiological study also showed that treatment with metformin was significantly associated with a $60 \%$ reduction in the risk of intrahepatic cholangiocarcinoma in diabetic patients (36). However, metformin did not show survival benefits for cholangiocarcinoma patients with diabetes in a recent investigation, but a very small number of patients were enrolled in this preliminary study (37). Metformin has been shown to increase cisplatin-induced cytotoxicity and inhibit ovarian and gastric tumor growth $(7,38)$. Cisplatin triggered activation of the AMPK pathway in glioma cells (38), and metformin enhanced the effect of cisplatin by inducing AMPK phosphorylation (7). In accordance, we showed here that metformin synergized with gemcitabine and cisplatin to induce apoptosis and inhibit the proliferation of cholangiocarcinoma cells by inducing the phosphorylation of AMPK, and sequential downregulation of cyclin D1. Gemcitabine and cisplatin are well known cytotoxic drugs used to treat a wide variety of cancers, and their combination chemotherapy was associated with a significant survival advantage for the treatment of patients with advanced biliary cancer (2). Gemcitabine inhibits the processes required for DNA synthesis (39), while cisplatin interacts with DNA to form DNA adducts, leading to the activation of apoptosis (40). Their combination has been shown to activate caspase-3 $(19,20)$, in accordance with our results that gemcitabine and cisplatin therapy markedly increased the apoptosis of cholangiocarcinoma cells by activating caspase- 3 .

In summary, conventional chemotherapeutic agents often lead to severe side-effects, such as damage to the intestine and hematologic suppression. However, metformin has exhibited only mild side-effects, such as abdominal discomfort, a metallic taste and mild anorexia, and these symptoms are reversible after dose reduction or discontinuation of the drug (41). Metformin has been demonstrated to be a very safe drug for over half a century since it was approved for diabetes treatment in 1957 (24). Therefore, combining metformin with gemcitabine and cisplatin may be advantageous, as metformin could be employed to enhance the anticancer activities and reduce the dose of chemotherapeutic agents to spare patients the side-effects without impairing the antitumor efficacy. Further investigation of this possibility is warranted.

\section{Acknowledgements}

The present study was supported by the Shandong Provincial Natural Scientific Research Foundation (BS2015YY024 and ZR2014HM099), the Shandong Provincial Scientific and Technology Development Program (2014GGB14041 and 2015GGB14242), and the Shandong Province Medical and Health Scientific and Technology Development Program (2014WS0093, 2014WS0095 and 2014WS0096).

\section{References}

1. Bridgewater J, Galle PR, Khan SA, Llovet JM, Park JW, Patel T, Pawlik TM and Gores GJ: Guidelines for the diagnosis and management of intrahepatic cholangiocarcinoma. J Hepatol 60: 1268-1289, 2014

2. Valle J, Wasan H, Palmer DH, Cunningham D, Anthoney A, Maraveyas A, Madhusudan S, Iveson T, Hughes S, Pereira SP, et al; $\mathrm{ABC}-02$ Trial Investigators: Cisplatin plus gemcitabine versus gemcitabine for biliary tract cancer. N Engl J Med 362: 1273-1281, 2010.

3. Ben Sahra I, Laurent K, Loubat A, Giorgetti-Peraldi S, Colosetti P, Auberger P, Tanti JF, Le Marchand-Brustel Y and Bost F: The antidiabetic drug metformin exerts an antitumoral effect in vitro and in vivo through a decrease of cyclin D1 level. Oncogene 27: 3576-3586, 2008. 
4. Simon D and Balkau B: Diabetes mellitus, hyperglycaemia and cancer. Diabetes Metab 36: 182-191, 2010.

5. Libby G, Donnelly LA, Donnan PT, Alessi DR, Morris AD and Evans JM: New users of metformin are at low risk of incident cancer: A cohort study among people with type 2 diabetes. Diabetes Care 32: 1620-1625, 2009.

6. He H, Ke R, Lin H, Ying Y, Liu D and Luo Z: Metformin, an old drug, brings a new era to cancer therapy. Cancer J 21: 70-74, 2015.

7. Yu G, Fang W, Xia T, Chen Y, Gao Y, Jiao X, Huang S, Wang J, $\mathrm{Li} \mathrm{Z}$ and Xie K: Metformin potentiates rapamycin and cisplatin in gastric cancer in mice. Oncotarget 6: 12748-12762, 2015.

8. Scotland S, Saland E, Skuli N, de Toni F, Boutzen H, Micklow E, Sénégas I, Peyraud R, Peyriga L, Théodoro F, et al: Mitochondrial energetic and AKT status mediate metabolic effects and apoptosis of metformin in human leukemic cells. Leukemia 27: 2129-2138, 2013.

9. Giannoni E, Taddei ML, Morandi A, Comito G, Calvani M, Bianchini F, Richichi B, Raugei G, Wong N, Tang D, et al: Targeting stromal-induced pyruvate kinase M2 nuclear translocation impairs oxphos and prostate cancer metastatic spread. Oncotarget 6: 24061-24074, 2015.

10. Fujihara S, Kato K, Morishita A, Iwama H, Nishioka T, Chiyo T, Nishiyama N, Miyoshi H, Kobayashi M, Kobara H, et al: Antidiabetic drug metformin inhibits esophageal adenocarcinoma cell proliferation in vitro and in vivo. Int $\mathrm{J}$ Oncol 46: 2172-2180, 2015.

11. Hsieh SC, Tsai JP, Yang SF, Tang MJ and Hsieh YH: Metformin inhibits the invasion of human hepatocellular carcinoma cells and enhances the chemosensitivity to sorafenib through a downregulation of the ERK/JNK-mediated NF- $\mathrm{BB}$-dependent pathway that reduces uPA and MMP-9 expression. Amino Acids 46: 2809-2822, 2014

12. Fujimori T, Kato K, Fujihara S, Iwama H, Yamashita $T$, Kobayashi K, Kamada H, Morishita A, Kobara H, Mori H, et al: Antitumor effect of metformin on cholangiocarcinoma: In vitro and in vivo studies. Oncol Rep 34: 2987-2996, 2015.

13. Soo JS, Ng CH, Tan SH, Malik RA, Teh YC, Tan BS, Ho GF, See MH, Taib NA, Yip CH, et al: Metformin synergizes 5-fluorouracil, epirubicin, and cyclophosphamide (FEC) combination therapy through impairing intracellular ATP production and DNA repair in breast cancer stem cells. Apoptosis 20: 1373-1387, 2015.

14. Honjo S, Ajani JA, Scott AW, Chen Q, Skinner HD, Stroehlein J, Johnson RL and Song S: Metformin sensitizes chemotherapy by targeting cancer stem cells and the mTOR pathway in esophageal cancer. Int J Oncol 45: 567-574, 2014.

15. Ling S, Feng T, Ke Q, Fan N, Li L, Li Z, Dong C, Wang C, $\mathrm{Xu}$ F, Li Y, et al: Metformin inhibits proliferation and enhances chemosensitivity of intrahepatic cholangiocarcinoma cell lines. Oncol Rep 31: 2611-2618, 2014.

16. He C, Dong X, Zhai B, Jiang X, Dong D, Li B, Jiang H, Xu S and Sun X: MiR-21 mediates sorafenib resistance of hepatocellular carcinoma cells by inhibiting autophagy via the PTEN/Akt pathway. Oncotarget 6: 28867-28881, 2015

17. Zhu H, Mi Y, Jiang X, Zhou X, Li R, Wei Z, Jiang H, Lu J and Sun $X$ : Hepatocyte nuclear factor 6 inhibits the growth and metastasis of cholangiocarcinoma cells by regulating miR-122. J Cancer Res Clin Oncol 142: 969-980, 2016.

18. Rizvi S and Gores GJ: Pathogenesis, diagnosis, and management of cholangiocarcinoma. Gastroenterology 145: 1215-1229, 2013.

19. Lee EK, Jinesh G G, Laing NM, Choi W, McConkey DJ and Kamat AM: A Smac mimetic augments the response of urothelial cancer cells to gemcitabine and cisplatin. Cancer Biol Ther 14: 812-822, 2013

20. Ma Y, Yu WD, Trump DL and Johnson CS: $1,25 D_{3}$ enhances antitumor activity of gemcitabine and cisplatin in human bladder cancer models. Cancer 116: 3294-3303, 2010.

21. Wang J, Ma Y, Jiang H, Zhu H, Liu L, Sun B, Pan S, Krissansen GW and Sun X: Overexpression of von Hippel-Lindau protein synergizes with doxorubicin to suppress hepatocellular carcinoma in mice. J Hepatol 55: 359-368, 2011.
22. Blechacz B, Komuta M, Roskams T and Gores GJ: Clinical diagnosis and staging of cholangiocarcinoma. Nat Rev Gastroenterol Hepatol 8: 512-522, 2011.

23. Erice O,Merino-Azpitarte M,Arbelaiz A, Gutierrez-Larranaga M, Jiménez-Agüero R, Perugorria MJ, Bujanda L and Banales JM: Molecular mechanisms of cholangiocarcinogenesis: New potential targets for therapy. Curr Drug Targets 16: 1, 2015.

24. Nattrass M and Alberti KG: Biguanides. Diabetologia 14: 71-74, 1978.

25. Pryor R and Cabreiro F: Repurposing metformin: An old drug with new tricks in its binding pockets. Biochem J 471: 307-322, 2015.

26. Vakana E, Altman JK and Platanias LC: Targeting AMPK in the treatment of malignancies. J Cell Biochem 113: 404-409, 2012.

27. Hardie DG, Ross FA and Hawley SA: AMP-activated protein kinase: A target for drugs both ancient and modern. Chem Biol 19: 1222-1236, 2012.

28. Liang J, Shao SH, Xu ZX, Hennessy B, Ding Z, Larrea M, Kondo S, Dumont DJ, Gutterman JU, Walker CL, et al: The energy sensing LKB1-AMPK pathway regulates $\mathrm{p} 27^{\mathrm{kipl}}$ phosphorylation mediating the decision to enter autophagy or apoptosis. Nat Cell Biol 9: 218-224, 2007.

29. Del Barco S, Vazquez-Martin A, Cufí S, Oliveras-Ferraros C, Bosch-Barrera J, Joven J, Martin-Castillo B and Menendez JA: Metformin: Multi-faceted protection against cancer. Oncotarget 2: 896-917, 2011.

30. Ding L, Liang G, Yao Z, Zhang J, Liu R, Chen H, Zhou Y, Wu H, Yang B and He Q: Metformin prevents cancer metastasis by inhibiting M2-like polarization of tumor associated macrophages. Oncotarget 6: 36441-36455, 2015

31. Rocha GZ, Dias MM, Ropelle ER, Osório-Costa F, Rossato FA, Vercesi AE, Saad MJ and Carvalheira JB: Metformin amplifies chemotherapy-induced AMPK activation and antitumoral growth. Clin Cancer Res 17: 3993-4005, 2011.

32. Kato K, Gong J, Iwama H, Kitanaka A, Tani J, Miyoshi H, Nomura K, Mimura S, Kobayashi M, Aritomo Y, et al: The antidiabetic drug metformin inhibits gastric cancer cell proliferation in vitro and in vivo. Mol Cancer Ther 11: 549-560, 2012.

33. Miyoshi H, Kato K, Iwama H, Maeda E, Sakamoto T, Fujita K, Toyota Y, Tani J, Nomura T, Mimura S, et al: Effect of the anti-diabetic drug metformin in hepatocellular carcinoma in vitro and in vivo. Int J Oncol 45: 322-332, 2014.

34. Musgrove EA, Caldon CE, Barraclough J, Stone A and Sutherland RL: Cyclin D as a therapeutic target in cancer. Nat Rev Cancer 11: 558-572, 2011.

35. Jiralerspong S, Palla SL, Giordano SH, Meric-Bernstam F, Liedtke C, Barnett CM, Hsu L, Hung MC, Hortobagyi GN and Gonzalez-Angulo AM: Metformin and pathologic complete responses to neoadjuvant chemotherapy in diabetic patients with breast cancer. J Clin Oncol 27: 3297-3302, 2009.

36. Chaiteerakij R, Yang JD, Harmsen WS, Slettedahl SW, Mettler TA, Fredericksen ZS, Kim WR, Gores GJ, Roberts RO, Olson JE, et al: Risk factors for intrahepatic cholangiocarcinoma: Association between metformin use and reduced cancer risk. Hepatology 57: 648-655, 2013.

37. Yang Z, Zhang X, Roberts RO, Roberts LR and Chaiteerakij R: Metformin does not improve survival of cholangiocarcinoma patients with diabetes. Hepatology 63: 667-668, 2016.

38. Rattan R, Graham RP, Maguire JL, Giri S and Shridhar V: Metformin suppresses ovarian cancer growth and metastasis with enhancement of cisplatin cytotoxicity in vivo. Neoplasia 13: 483-491, 2011.

39. de Sousa Cavalcante L and Monteiro G: Gemcitabine: Metabolism and molecular mechanisms of action, sensitivity and chemoresistance in pancreatic cancer. Eur J Pharmacol 741: 8-16, 2014.

40. Dasari S and Tchounwou PB: Cisplatin in cancer therapy: Molecular mechanisms of action. Eur J Pharmacol 740: 364-378, 2014.

41. Bailey CJ and Turner RC: Metformin. N Engl J Med 334: 574-579, 1996. 